\title{
Алгоритм работы контроллера управления электромагнитным проиессом в длинной линии
}

\author{
B.B. Аъготчиков 1, д.m.н., npoфpeccop, vvldrive@yandex.ru \\ B.Н. Денисов ${ }^{1}$, д.m.н., nрофрессор, VM@sbmpei.ru \\ T.C. Марькина ${ }^{1,}$, аспирант, tatyana.larkina.2015@yandex.ru \\ 1 Смоленский филиал Наиионального исследовательского университета МЭИ, \\ 2. Смоленск, 214013, Россия
}

В статье рассматривается вопрос управления процессом выделения мощности в заданном сечении вторичного тела от токов, наведенных индуктором. Данное управление может быть применено в ряде технологий дефектоскопии, нагрева массивных объектов с помощью индукционных токов. Индуктор содержит управляемые источники напряжения, а система управления процессом должна обеспечивать возможность идентификации параметров объекта воздействия (вторичного тела) с целью фиксации и оценки эффективности результата энергетического воздействия.

Для математического описания электромагнитных процессов в индукторе используется представление объекта длинной линией с распределенными параметрами. Математический аппарат, выбранный для решения задачи, предусматривает замену переменной с целью обнуления граничных условий. Распределение модуля напряжения вдоль линии в зависимости от времени найдено с использованием разложения напряжения, введенных новых переменных в ряд Фурье, с целым числом полуволн нечетной синусоидальной функции. Определяется баланс по амплитудам дискретного и непрерывного разложений в ряд на выбранном разработчиком интервале рабочих частот. Принимается во внимание соотношение частота источника напряжения-длина волны напряжения вдоль линии.

В алгоритме реализуется контроль точности в представлении распределения напряжения вдоль длинной линии в процессе решения задачи с возможностью использования в качестве управляющего устройства микроконтроллера. Рост числа элементов ряда ведет к усложнению ПО контроллера, управляющего источниками. Зная длину линии и требуемую точность, можно оптимально управлять числом ряда разложения, меняя частоту источников. Решение задачи и тест алгоритма проведены с использованием математического пакета Мaple. Результат иллюстрируется численным примером поиска распределения напряжения с заданным рассогласованием напряжений на концах линии по фазе, частоте и амплитуде отдельно. Приведенное математическое обеспечение является составной частью ПО контроллера управления технологическим процессом.

Ключевые слова: длинная линия, распределенные сопротивления, граничные условия, ряд Фурье, микроконтроллер, программное обеспечение.

Технологический процесс передачи мощности от индуктора к вторичному телу с укрупненной блок-схемой ПО представлен на рисунке 1 . В массиве вторичного тела (верхняя часть рисунка) необходимо обеспечить управляемый процесс позиционирования области выделения максимальной мощности, например, мощности разогрева вторичного тела токами Фуко [1-5]. Для наглядности показана пиктограмма перемещения экстремума мощности $(P)$ вдоль линейной координаты $(x)$. В первичном контуре (индукторе) этим максимумам мощности соответствуют пучности напряжения $u(x, t)$ в длинной линии. Энергия в индуктор поступает от двух управляемых источников напряжения $E_{1}(t)$ и $E_{2}(t)$ с управляемым вектором: амплитуда $(A)$, частота $(\omega)$

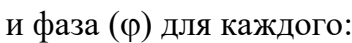

$$
\left\{\begin{array}{l}
A_{1}, \omega_{1}, \varphi_{1} ; \\
A_{2}, \omega_{2}, \varphi_{2} .
\end{array}\right.
$$

В качестве отклика объекта на энергетическое воздействие контроллером воспринимается вектор напряжений и токов в конце и начале линии (длина линии $l$, источник не идеален - имеет внутреннее сопротивление):

$$
\left\{\begin{array}{l}
u(0, t), i(0, t) ; \\
u(l, t), i(l, t) .
\end{array}\right.
$$

Векторы (2) используются для оценки качества энергетического воздействия на вторичное тело через идентификацию его параметров (блок 4 ПО микроконтроллера $(\mu C)$, рис. 1). Кроме того, ПО $\mu C$ включает три блока (1-3) с традиционными функциями организации алгоритма управления: инициализации аппаратных средств $\mu C$, выработки стратегии управления и математической модели процесса. Рассмотрим подробно математическую модель, положенную в основу ПО блока 3 (рис. 1).

Как известно, телеграфные уравнения описывают электромагнитные процессы во времени $(t)$ и пространстве $(x)$ в длинных линиях - линиях с распределенными параметрами [6-9]. Решение уравнений находят во временной области с использованием разложения в ряд Фурье напряжений источников на концах линии и математического аппарата комплексных переменных. Есть трудности в учете начальных условий на концах линии, в том числе и при использовании прямого решения дифференциальных уравнений двух переменных во временной области [10-13]. Предлагается метод решения телеграфных уравнений для двух регулируемых источников напряжения на концах линии. При решении уравнений предполагается возможность управления точностью процесса вычислений. В этом смысле метод близок к численным про- 


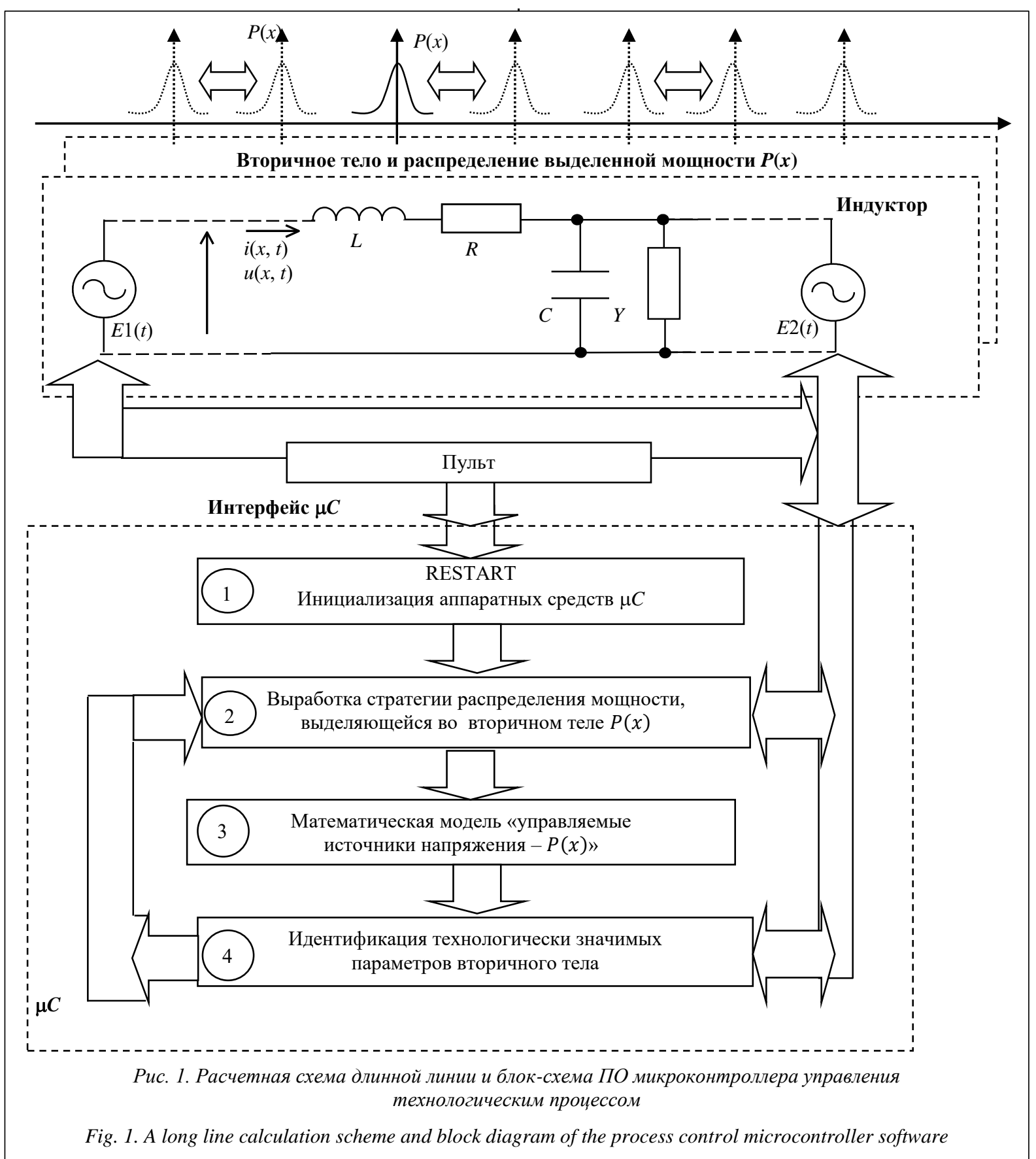

цедурам и хорошо адаптируется к средствам управления, выполненным на базе микроконтроллера.

Расчетная схема для линейного фрагмента $d x$ длинной линии $l$ изображена на рисунке 1 в блоке «индуктор». Через $R, L, C, Y$ обозначены, соответственно, распределенные активное сопротивление, индуктивность, емкость и проводимость, $i(x, t)$ и $u(x, t)$ - ток и напряжение.

Управление положением пучностей и узлов излучения планируется осуществлять двумя управляемыми источниками напряжения $E_{1}(t)$ и $E_{2}(t)$, подключенными к концам линии:

$$
\left\{\begin{array}{l}
E_{1}(t)=A_{1} \cdot \sin \left(\omega_{1} t_{1}+\varphi_{1}\right) ; \\
E_{2}(t)=A_{2} \cdot \sin \left(\omega_{2} t_{2}+\varphi_{2}\right) .
\end{array}\right.
$$

Законы изменения напряжения на источниках взаимозависимы, формируются микроконтроллером $\mu C$ при сопоставлении технологического задания (местоположение узлов и пучностей излучения - блок 2 (рис. 1)) и управляемых характеристик источников напряжения - их амплитуд $\left(A_{1}, A_{2}\right)$, частот $\left(\omega_{1}, \omega_{2}\right)$ и фаз $\left(\varphi_{1}, \varphi_{2}\right)$.

Таким образом, систему регулирования положения пучностей и узлов излучения можно определить как систему регулирования по управляющему воздействию без обратных связей. Вектор обратных связей (2) используется на этапе анализа результата энергетического воздействия на вторичное тело. 
Адаптируем исходную запись системы телеграфных уравнений к поставленной задаче управления:

$$
\left\{\begin{array}{l}
\frac{\partial u}{\partial x}=L \frac{\partial i}{\partial t}+R i \\
\frac{\partial i}{\partial x}=C \frac{\partial u}{\partial t}+Y u
\end{array}\right.
$$

Рассмотрим решение для самого общего случая - наличие двух управляемых источников напряжения на концах линии с оригинальными характеристиками. Тогда решение дифференциальных уравнений будет состоять из двух частей: свободной и вынужденной. В системе (4) продифференцируем первое из уравнений по переменной $x$, второе - по переменной $t$ и подставим в левую часть первого выражения значение производной $\frac{\partial i}{\partial x \partial t}$ из второго:

$$
u_{\mathrm{xx}}{ }^{\prime \prime}-L C u_{t t}^{\prime \prime}-(L Y+R C) \cdot u_{t}^{\prime}-R Y=0 .
$$

Для компактности записи порядок производной переменных в (5) показан верхним индексом, а независимые переменные, по которым берется дифференциал, - нижним. Начальные и конечные условия по положению и времени для напряжения в линии зададим системой уравнений:

$$
\left\{\begin{array}{l}
u(x, 0)=0 ; \\
u_{t}^{\prime}(x, 0)=0 ; \\
u(0, t)=E_{1}(t) ; \\
u(l, t)=E_{2}(t) .
\end{array}\right.
$$

Для получения решения задачи (5), (6) в виде ряда целых полуволн гармоник синусоидальной функции произведем замену переменной в (5), (6) с целью обнуления краевых условий на концах линии. Новую переменную обозначим $\tilde{u}$. Замена переменных имеет вид

$$
u=\tilde{u}+E_{1}(t) \frac{l-x}{l}+E_{2}(t) \frac{x}{l} .
$$

Очевидно, что $\widetilde{u}(0, t)=0, \tilde{u}(l, t)=0$.

Используя (5), подготовим массив частных производных для $\widetilde{u}$ :

$$
\left\{\begin{array}{l}
u_{x}^{\prime}=\tilde{u}_{x}^{\prime}+E_{1}(t) \frac{-1}{l}+E_{2}(t) \frac{1}{l} ; \\
u_{x x}^{\prime}=\tilde{u}_{x x}^{\prime}+0+0 ; \\
u_{t}^{\prime}=\tilde{u}_{t}^{\prime}+E_{1 t}^{\prime}(t) \frac{l-x}{l}+E_{2 t}^{\prime}(t) \frac{x}{l} ; \\
u_{t t}^{\prime}=\tilde{u}_{t t}^{\prime}+E_{1 t t}^{\prime}(t) \frac{l-x}{l}+E_{2 t t}^{\prime}(t) \frac{x}{l} .
\end{array}\right.
$$

Используя (3), (4) и (6), получим уравнение для новой переменной $\tilde{u}$, решение которого можно будет искать в виде разложения в ряд Фурье по собственным функциям однородной краевой задачи [14]:

$$
\begin{aligned}
& \tilde{u}_{x x}^{\prime \prime}-L C \tilde{u}_{t t}^{\prime \prime}-L C \tilde{E}_{t t}^{\prime \prime}-(L Y+R C) \tilde{u}_{t}^{\prime}- \\
& -(L Y+R C) \tilde{E}_{t}^{\prime}-R Y \tilde{u}-R Y \tilde{E},
\end{aligned}
$$

где используется обозначение $\tilde{E}=E_{1}(t) \frac{l-x}{l}+E_{2}(t) \frac{x}{l}$; назначение верхних и нижних индексов прежнее.

Принимая во внимание (6) и (7), могут быть получены начальные и краевые условия для новой переменной $\tilde{u}$ :

$$
\left\{\begin{array}{l}
u(x, 0)=\widetilde{u}(x, 0)+\left.\widetilde{E}\right|_{t=0}=0 ; \\
u_{t}^{\prime}(x, 0)=\widetilde{u}_{t}^{\prime}(x, 0)+\left.\widetilde{E}_{t}^{\prime}\right|_{t=0}=0 ; \\
\widetilde{u}(0, t)=0 ; \\
\widetilde{u}(l, t)=0 .
\end{array}\right.
$$

Как было отмечено, решение в поле новой переменной ищется в форме

$$
\tilde{u}(x, t)=\sum_{n=0}^{\infty} T_{n}(t) \sin \frac{\pi n x}{l},
$$

где $n$ - номер гармоники (целое число); $T_{n}(t)$ - изменение во времени амплитуды гармоники номер $n ; \sin \frac{\pi n x}{l}-$ собственные функции.

Переходя к новой переменной, подставим (11) в (9) и получим

$$
\begin{aligned}
& \sum_{n=0}^{\infty} F\left(T_{n}(t)\right) \sin \frac{\pi n x}{l}=L C\left(E_{1 t t}^{\prime \prime}(t) \frac{l-x}{l}+\right. \\
& \left.+E_{2 t t}^{\prime \prime}(t) \frac{x}{l}\right)+(L Y+R C)\left(E_{1 t}^{\prime}(t) \frac{l-x}{l}+\right. \\
& \left.+E_{2 t}^{\prime}(t) \frac{x}{l}\right)+R Y\left(E_{1}(t) \frac{l-x}{l}+E_{2}^{\prime}(t) \frac{x}{l}\right),
\end{aligned}
$$

где в левой части равенства введено обозначение

$$
\begin{aligned}
& F\left(T_{n}(t)\right)=-T_{n}(t)\left(\left(\frac{\pi n}{l}\right)^{2}+R Y\right)- \\
& -L C\left(T_{n}(t)\right)_{t t}^{\prime \prime}-(L Y+R C)\left(T_{n}(t)\right)_{t}^{\prime} .
\end{aligned}
$$

Левая часть выражения (12) будет равна правой части, если амплитуда каждой из гармоник левой части этого выражения будет равна амплитуде соответствующей гармоники правой части. Разложим правую часть выражения (12) в ряд Фурье с ядром преобразования: $\sin \frac{\pi n x}{l}$. Тогда баланс для (12) записывается так:

$$
\begin{aligned}
& \sum_{n=0}^{\infty}\left[-a\left(T_{n}(t)\right)_{t t}^{\prime \prime}-b\left(T_{n}(t)\right)_{t}^{\prime}-d\left(T_{n}(t)\right)\right]= \\
& =\frac{2}{l} \int_{0}^{l}\left[a\left(E_{1 t t}^{\prime \prime}(t) \frac{l-x}{l}+E_{2 t t}^{\prime \prime}(t) \frac{x}{l}\right)+\right. \\
& +b\left(E_{1 t}^{\prime}(t) \frac{l-x}{l}+E_{2 t}^{\prime}(t) \frac{x}{l}\right)+ \\
& \left.+c\left(E_{1}(t) \frac{l-x}{l}+E_{2}^{\prime}(t) \frac{x}{l}\right)\right] d x,
\end{aligned}
$$

где $a=L C ; b=L Y+R C ; c=R Y ; d=\left(\frac{n \pi}{l}\right)^{2}+R Y$. 


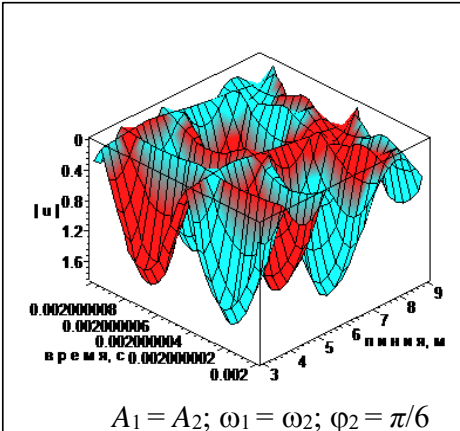

a)

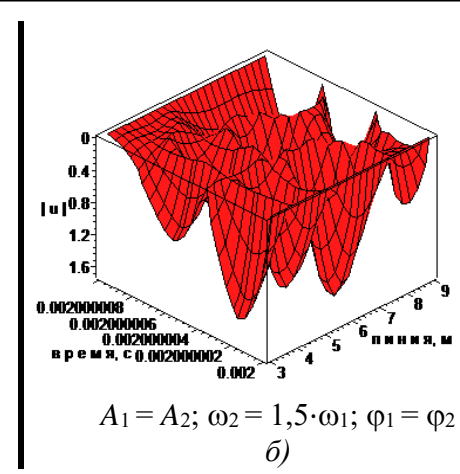

б)

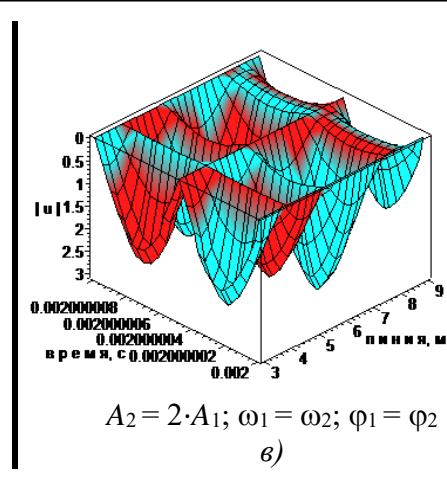

B)

Рис. 2. Распределение модуля и фазы напряжения (интенсивность цзвета) в длинной линии при несогласованности фаз питающчих напряжений (а), их частот (б) или амплитуд (в)

Fig. 2. The module and voltage phase distribution (color intensity) in a long line when supply phases mismatch (a), their frequencies (6) or amplitudes ( 6 )

Упростить выражение (14) можно, взяв интеграл в правой части, учитывая, что производные по времени питающих напряжений не зависят от $x$. При этом вычисление определенных интегралов, как и дифференциалов, не вызывает затруднений. Приведя подобные, получим:

$$
\begin{aligned}
& \sum_{n=0}^{\infty}\left[a\left(T_{n}(t)\right)_{t t}^{\prime \prime}+b\left(T_{n}(t)\right)_{t}^{\prime}+d\left(T_{n}(t)\right)\right]= \\
& =\left(A_{1} a \omega_{1}^{2}-A_{1} c\right) \cdot \sin \left(\omega_{1} t+\varphi_{1}\right)- \\
& -A_{1} b \omega_{1} \cos \left(\omega_{1} t+\varphi_{1}\right)+\left(A_{2} a \omega_{2}^{2}-A_{2} c\right) \sin \left(\omega_{2} t+\varphi_{2}\right)- \\
& -A_{2} b \omega_{2} \cos \left(\omega_{2} t+\varphi 2\right) .
\end{aligned}
$$

Алгоритм поиска распределения напряжения вдоль длинной линии состоит из нескольких шагов.

1. Для известных характеристик линии и подключенных источников напряжения рассчитываются по (15) амплитуды гармоник сигнала $\left.T_{n}(t)\right|_{n=0 \ldots \infty}$. Число гармоник ограничивается требуемой точностью, балансом мощностей источников и передаваемого в линию сигнала или другими критериями.

2. По (11) определяется выражение для новой (вспомогательной) переменной. При этом постоянные интегрирования в (15) определяются из начальных условий (10).

3. Используя (7), ищется распределение напряжения по длине линии.

Три данных шага алгоритма математических расчетов дают базу для формирования итерационной процедуры поиска нужного распределения пучностей напряжения вдоль длинной линии (содержание блока 3 ПО $\mu C$, рис. 1).

В качестве иллюстрации предложен расчет модуля напряжения в длинной линии (рис. 2) со следующими параметрами: $l=9 \mathrm{M} ; R=10^{-3} \mathrm{OM} / \mathrm{M}$; $L=10^{-6} \Gamma \mathrm{H} / \mathrm{M} ; C=5 \cdot 10^{-11} \Phi / \mathrm{M} ; Y=3 \cdot 10^{-6} \mathrm{CM} / \mathrm{M}$; $A_{1}=1 \mathrm{~B} ; \omega_{1}=2 \cdot \pi \cdot 100^{6} \mathrm{c}^{-1} ; \varphi_{1}=0$.

Пример был решен с использованием пакета символьной математики Maple для пяти первых членов ряда (11): $n=5$. На рисунке 2 изменение фазы модуля напряжения вдоль линии в функции времени показано модуляцией цвета: заметна деформация картины распределения модуля напряжения вдоль линии в функции времени, для поверхности (б) характерно нарастающее увеличение аргумента, что находит отражение в монохромности рисунка. Частота напряжения верно соответствует числу полупериодов пучностей, укладывающихся по длине линии.

Предложенный аналитический метод решения телеграфных уравнений может быть востребован в случаях наличия управляемых источников на концах линии и появляющейся возможности программного задания местоположения узлов и пучностей напряжения вдоль линии. Математическую модель предполагается использовать, например, в установках местного прогрева вторичного тела с индуктором, в дефектоскопии.

\section{Литература}

1. Sordiashie E. Electromagnetic harvesting to power energy management sensors in the built environment. Thesis. Nebraska, 2012, $113 \mathrm{p}$.

2. Mardiguian M. Simple method for predicting a cable shielding factor based on transfer impedance. EMC Directory \& design guide: Interference Technology, Plymouth Meeting. Pennsylvania, 2012, pp. 1-5.

3. Ahmadi H., Mohseni S., Shayegani Akmal A.A. Electromagnetic fields near transmission lines - problems and solutions. Iran. J. Environ. Health. Sci. Eng., 2010, vol. 7, no. 2, pp. 181-188.

4. Rudnev V., Loveless D., Cook R. Handbook of Induction Heating. 2nd Ed. Taylor \& Francis Group, LLC, 2017, 719 p.

5. Нефедов В.И., Хахин В.И., Битюков В.К. и др. Метрология и радиоизмерения; [под ред. В.И. Нефедова]. М.: Высш. школа, 2006. $526 \mathrm{c}$.

6. Атабеков Г.И. Основы теории цепей. СПб: Лань, 2009. $432 \mathrm{c}$.

7. Корн Г.А. Справочник по математике (для научных работников и инженеров). Определения, теоремы, формулы; [пер. с англ.]. СПб: Лань, 2003. 831 c.

8. Бессонов Л.А. Теоретические основы электротехники. Электрические цепи. М.: Гардарики, 2006. 701 с.

9. Улахович Д.А. Основы теории линейных электрических цепей. СПб: БХВ-Петербург, 2009. 816 с.

10. Smith P.W. Transient electronics: Pulsed circuit technology. Chichester: John Wiley \& Sons, 2002, 272 p.

11. Карлов Н.В. Колебания, волны, структуры. М.: Физматлит, 2008. $496 \mathrm{c}$. 
12. Тамм И.Е. Основы теории электричества. М.: Наука, 2003. $504 \mathrm{c}$

13. Кудряшов Ю.Б., Петров Ю.Ф., Рубин А.Б. Радиационная биофизика: радиочастотные и микроволновые электромаг- нитные излучения. М.: Физматлит, 2008. 184 с.

14. Эдвардс Ч.Г., Пенни Д.Э. Дифференциальные уравнения и краевые задачи. Моделирование и вычисление с помощью Mathematica, Maple и MATLAB. М.: Вильямс, 2008. 1104 c.
Software \& Systems

DOI: $10.15827 / 0236-235 X .124 .798-802$
Received 29.06.18

2018, vol. 31, no. 4, pp. 798-802

\section{Algorithm of the controller of electromagnetic process control in a long line}

V.V. Lgotchikov ${ }^{1}$, Dr.Sc. (Engineering), Professor, vvldrive@yandex.ru

V.N. Denisov ${ }^{1}$, Dr.Sc. (Engineering),Professor,VM@sbmpei.ru

T.S. Larkina ${ }^{1}$, Postgraduate Student, tatyana.larkina.2015@yandex.ru

${ }^{1}$ Smolensk Branch of the Moscow Power Engineering Institute, Smolensk, 214013, Russian Federation

Abstract. The paper discusses the issue of controlling the process of power allocation in a given secondary body cross section from currents induced by an inductor. This control can be applied in a number of flaw detection technologies, heating massive objects using induction currents. The inductor contains controlled voltage sources. The process control system must provide the ability to identify impact object (secondary body) parameters in order to fix and evaluate the effectiveness of the energy impact result.

For mathematical description of electromagnetic processes in the inductor, the authors use an object representation by a long line with distributed parameters. The selected mathematical apparatus provides replacement of a variable in order to zero the boundary conditions. The voltage modulus is distributed along the line depending on time using the voltage expansion, introduction of new variables into a Fourier series with an integer number of half-waves of an odd sinusoidal function. The authors determine the balance by the amplitudes of discrete and continuous expansion in a row on a working frequency interval selected by the developer. They take into account the ratio of the voltage source frequency - the voltage wavelength along the line.

The algorithm implements accuracy control in the representation of voltage distribution along a long line when solving a problem with the possibility of using it as a microcontroller control device. The increase in the number of series elements leads to complication of controller software that controls sources. If the line length and the required accuracy is known, it is possible to control the number of the expansion series optimally by varying source frequency.

The authors used the Maple mathematical package to solve the problem and test the algorithm. The result is illustrated by a numerical example of a voltage distribution search with a specified voltage mismatch at line ends in phase, frequency and amplitude separately. The provided software is an integral part of the process control controller software.

Keywords: long line, distributed resistances, border conditions, the Fourier series, microcontroller, software.

\section{References}

1. Sordiashie E. Electromagnetic Harvesting to Power Energy Management Sensors in the Built Environment. Thesis. Nebraska, 2012, 113 p.

2. Mardiguian M. Simple method for predicting a cable shielding factor based on transfer impedance. EMC Directory \& Design Guide: Interference Technology, Plymouth Meeting. Pennsylvania, 2012, pp. 1-5.

3. Ahmadi H., Mohseni S., Shayegani Akmal A.A. Electromagnetic fields near transmission lines - problems and solutions. Iran. J. Environ. Health. Sci. Eng. 2010, vol. 7, no. 2, pp. 181-188.

4. Rudnev V., Loveless D., Cook R. Handbook of Induction Heating. 2nd ed. Taylor \& Francis Group, LLC, 2017, $719 \mathrm{p}$.

5. Nefedov V.I., Khakhin V.I., Bityukov V.K., Sigov A.S. Metrology and Radio Measurements. Moscow, Vysshaya shkola Publ., 2006, 526 p.

6. Atabekov G.I. Fundamentals of the Theory of Chains. St. Petersburg, Lan Publ., 2009, 432 p.

7. Korn Gr.A., Korn Th.M. Mathematical Handbook for Scientists and Engineers. McGraw-Hill Publ., 1968, 1130 p. (Russ. ed.: St. Petersburg, Lan Publ., 2003, 831 p.).

8. Bessonov L.A. Theoretical Foundations of Electrical Engineering. Electrical Circuits. Moscow, Gardariki Publ., 2006, $701 \mathrm{p}$.

9. Ulakhovich D.A. Fundamentals of the Theory of Linear Electric Circuits. St. Petersburg, BHV-Peterburg Publ., 2009, $816 \mathrm{p}$.

10. Smith P.W. Transient Electronics: Pulsed Circuit Technology. Chichester, John Wiley \& Sons Publ., 2002, 272 p.

11. Karlov N.V. Oscillations, Waves, Structures. Moscow, Fizmatlit Publ., 2008, 496 p.

12. Tamm I.E. Fundamentals of the Theory of Electricity. Moscow, Nauka Publ., 2003, 504 p.

13. Kudryashov Yu.B., Petrov Yu.F., Rubin A.B. Radiation Biophysics: Radio-Frequency and Microwave Electromagnetic Radiation. Moscow, Fizmatlit Publ., 2008, 184 p.

14. Edvards Ch.G., Penni D.E. Differential Equations and Boundary Value Problems. Modeling and Computation with Mathematica, Maple and MATLAB. Moscow, Vilyams Publ., 2008, 1104 p. 